\title{
FACTORES QUE INFLUYEN EN EL HÁBITO DE FUMAR EN ESTUDIANTES DE LA FACULTAD DE ENFERMERÍA DE LA UNIVERSIDAD NACIONAL SAN LUIS GONZAGA DE ICA, OCTUBRE 2012 - OCTUBRE 2013
}

\section{Factors in smoking in students of nursing faculty of Universidad Nacional San Luis Gonzaga Ica,} October 2012 - October 2013

Isabel Natividad Urure Velazco ${ }^{1,2, a, b, f}$, Rosario Campos Soto ${ }^{1,2, a, c, d}$, Cecilia Teresa Ventura Miranda ${ }^{1,4, a, b, e}$, Yrma Angélica Ríos Del Águila ${ }^{1,3, a, b, d}$, María Cristina Escate Quijandría ${ }^{1, g}$

${ }^{1}$ Facultad de Enfermería. Universidad Nacional San Luis Gonzaga de Ica. Ica, Perú

${ }^{2}$ Hospital Santa María del Socorro. Ica, Perú.

${ }^{3}$ Hospital Augusto Hernández Mendoza. Ica, Perú

${ }^{4}$ Hospital Regional de Ica, Ica, Perú

${ }^{a}$ Licenciada en Enfermería, ${ }^{b}$ Enfermera Especialista de Cirugía General, ${ }^{c}$ Enfermera Especialista en cuidado del niño, ${ }^{d}$ Magister en Salud Pública, ${ }^{\mathrm{e}}$ Magister en Ciencias de la Enfermería, ${ }^{\mathrm{f} D o c t o r}$ en Ciencias de la Salud, ${ }^{\mathrm{g}}$ Estudiante de Enfermería.

\section{RESUMEN}

Objetivo: Identificar los factores que influyen en el hábito de fumar en estudiantes de la Facultad de Enfermería de la Universidad Nacional "San Luis Gonzaga" de Ica. Material y métodos: Estudio descriptivo - transversal. Constituido por 143 estudiantes del I al X Ciclo. Haciendo uso de un cuestionario validado de 12 reactivos de opción múltiples y dicotómicas. Resultados: Factores socioculturales: El motivo que indujo a fumar fue por curiosidad $58 \%$, por presión de grupo 16,8\%, considerarse independiente $7,0 \%$, por presencia de fumadores en familia 4,2\%. Factores económicos: $84,6 \%$ gastan menos de $S / 5$ nuevos soles, y $15,4 \%$ gastan más de $S / 5$ Nuevos soles con un monto anual para la compra de cigarrillos $84,6 \%$, y $22,4 \%$ semanal o mensual. Factores personales: Consumo de drogas es muy bajo con 1,5\%, siendo estos los que fuman ocasionalmente. Respecto al consumo de alcohol el $24,5 \%$ beben licor y en su mayoría son los que fuman cigarrillos. Falta de práctica del deporte constituye $44,1 \%$, por conflictos familiares y fracaso o abandono familiar el $30,1 \%$ y $25,4 \%$ respectivamente. Grupo etáreo predominante fue entre $16-$ 20 años el $62,2 \%$, sexo femenino mayoritariamente con $79,7 \%$, edad de inicio fue de 15 años a más con $65 \%$ y en menor porcentaje respondieron que iniciaron a los 10 años con $3,5 \%$. Frecuencia de fumar fue ocasional $62,2 \%$. El 26,93\% tienen el hábito de fumar (143). Conclusiones: Los factores socioculturales, económicos y personales influyen en el hábito de fumar en estudiantes de la Facultad de Enfermería de la Universidad Nacional San Luis Gonzaga de Ica.

PALABRAS CLAVE: Factores, hábito, fumar, estudiantes de enfermería.

\section{SUMMARY}

Objective: To identify factors that influence smoking among students of the Faculty of Nursing of the Universidad Nacional San Luis Gonzaga de Ica. Methods: Descriptive study - cross. Consisting of 143 students from I to X Cycle. Using a validated questionnaire of 12 items of multiple choice and dichotomous. Results: Socio-cultural factors: The reason that induced by smoking was $58 \%$ curiosity, peer pressure by $16.8 \%, 7.0 \%$ considered independent by the presence of $4.2 \%$ family smoking. Economic factors: $84.6 \%$ spend less than 5 new soles, and $15.4 \%$ spending more 5 new 
soles, with an annual amount for the purchase of cigarettes $846 \%$, and $224 \%$ weekly or monthly. Personal factors: Drug use is very low at $1.5 \%$, and those who smoke occasionally. Regarding alcohol $24.5 \%$ drink liquor and are mostly those who smoke cigarettes. Lack of sport constitutes $44.1 \%$, and failure by family conflicts or family abandonment $30.1 \%$ and $25.4 \%$ respectively. Predominant age group was between $16-20$ years, $62.2 \%$ female majority with $79.7 \%$, age of onset was 15 years and $65 \%$ more with a lesser percentage responded that began at age 10 with $3.5 \%$. Occasional smoking rate was $62.2 \%$. The $26.93 \%(143)$ had smoking. Conclusions: Socio-cultural, economic and personal factors influence smoking among students of the Faculty of Nursing of the Universidad Nacional San Luis Gonzaga de Ica.

KEYWORDS: factors, habit, smoking, nursing students.

\section{INTRODUCCIÓN}

El consumo del tabaco en el mundo por su gran magnitud es un problema de salud pública, pues las cifras muestran que el $29 \%$ de la población mundial fuma (1), y está bien documentado como la principal causa evitable de morbilidad y mortalidad en el mundo.

El tabaco es la segunda droga más consumida en el mundo, existen muchas evidencias indicando que fumar ocasiona cáncer de pulmón, enfisema pulmonar, etc. Las consecuencias también se presentan, aunque en menor magnitud, sobre los fumadores pasivos (2).

Es importante considerar que el tabaco no es peligroso solo para quienes lo consumen, en forma de cigarrillo fumado, sino para quienes inhalan las sustancias procedentes de él a través del humo del tabaco medioambiental(3), hay denominado humo de segunda mano (SHS), el cual es reconocido como causante de numerosas patologías en personas no fumadoras, expuestas medioambientalmente.

En los Estados Unidos de América, se ha reportado que el riesgo de fumar es mayor cuando coinciden otros factores como padres o pertenecer a hogares de bajo ingresos económicos o bajo nivel educativo. Entre los factores sociales asociados con el inicio del tabaquismo en la adolescencia está la presión psicosocial de los amigos, tener una imagen social deseada, facilidades para esclarecer relaciones interpersonales, las mujeres utilizan como un medio para controlar su peso, y en algunos adolescentes para reafirmar el paso de la niñez a la adultez (4). Asimismo hay datos prospectivos sobre el consumo del uso del tabaco, hallándose que el comienzo del hábito de fumar está determinado por un conjunto de factores socioculturales, económicos y familiares.

En estudios internacionales sobre prevalencia de fumadores en estudiantes de Medicina de la Universidad Complutense de Madrid (UCM) en el año 2002 fue de $24,3 \%$ y de ex fumador de $8,1 \%$ y en estudiantes del área de la salud de la Universidad del Valle, y Colombia, en el año 2003 la prevalencia de fumador activo fue de $11,5 \%$ y de exfumador $26,3 \%(2)$.

En el Perú, aproximadamente dos terceras partes de la población general ha consumido 
cigarrillos alguna vez en su vida(5), y a pesar de que las investigaciones realizadas en la población, principalmente escolar y familiar, la información epidemiológica sobre el consumo del tabaco en peruanos profesionales de las ciencias de la salud es escasa.

El $32,2 \%$ de los estudiantes universitarios en Lima fuma al menos una vez a la semana(6).En estudios realizados por Zárate et al año 2002 sobre prácticas de consumo de tabaco y otras drogas en estudiantes de ciencias de la salud en una universidad privada de Lima, Perú, se halló que el 45,3\% consume tabaco desde los 16 años, 25\% fuma por primera vez en una fiesta y $45,8 \%$ sigue fumando en eventos sociales(7). A nivel local en el Departamento de Ica, Castillo R. et al, concluyeron que hay una prevalencia del consumo de tabaco en los alumnos de la Institución Educativa de 12,68\% identificándose como factores de riesgo prevalentes, el inicio de consumo a los 15 años de edad y como motivo de inicio de consumo el ser aceptado por el grupo(8).

Actualmente la Organización Mundial de la Salud (OMS) promueve en forma prioritaria que el personal médico y paramédico deje de fumar porque estos desempeñan una función importante como educadores en la prevención de comportamientos saludables en la población en general, por la influencia de las/os enfermeros y médicos en programas de cesación de consumo de tabaco en sus pacientes(9).

Los hábitos del consumo del tabaco en estudiantes universitarios del área de salud y en nuestro medio local (Ica) son poco conocidos pero importantes por su impacto en la formación de recursos que prestarán a la población expuesta a este grave problema de salud pública. Este estudio se realizó con el objetivo de delimitar los factores que influyen en el hábito de fumar en estudiantes de la Facultad de Enfermería de la Universidad Nacional San Luis Gonzaga de Ica, con el propósito de contribuir al diagnóstico de su consumo de tabaco e incentivar al desarrollo de programas de prevención e intervención.

\section{MATERIAL Y MÉTODOS}

El estudio utilizó el método descriptivo de corte transversal ejecutándose en la Facultad de Enfermería de la Universidad Nacional San Luis Gonzaga de Ica, que se encuentra ubicada en la Ciudad Universitaria Panamericana Sur Km 305. Av. Los Maestros $\mathrm{S} / \mathrm{N}$, Ica - Perú, con una población estudiantil en la Facultad de Enfermería de 531 estudiantes. Muestra constituida de 143 estudiantes del I al X Ciclo. Obteniéndose este tamaño muestral por una fórmula para población finita.

Se excluyeron los estudiantes que estuvieron realizando prácticas pre-profesionales fuera de Ica. Se hizo uso de un cuestionario que constó de 2 partes: Primera parte: Datos generales (características demográficas): edad, ciclo, estado civil, sexo, lugar de procedencia, otros relacionados a: con qué frecuencia fuma, a qué edad inició su hábito de fumar; segunda parte: con reactivos referente a los factores socioculturales, económicos y personales que influyen en el hábito de fumar, se utilizó 12 reactivos. 
En la recolección de datos se utilizó como técnica la encuesta y como técnica el cuestionario elaborado por las autoras, previamente validado por juicio de expertos y reestructurado con preguntas de opción múltiple y dicotómicas (si, no). Para evaluar la confiabilidad del instrumento se realizó una prueba piloto al $15 \%$ de la muestra en estudio (no incluyéndose los 143 estudiantes), los que permitieron determinar las características finales del instrumento. Se hizo uso del coeficiente de confiabilidad alfa Cronbach, obteniéndose un valor 0,76 , se utilizó el programa de análisis de datos SPSS.

\section{RESULTADOS.}

Referente a los factores socioculturales: EI motivo que les indujo a fumar fue por curiosidad 5,8\% (83 alumnos), presión de grupo $16,8 \%$ (24alumnos), por independencia, presencia de fumadores constituyó un 7\% (10alumnos) y 4,2\% (6alumnos) respectivamente.

Factores económicos: En su mayoría gastan menos de 5.00 Nuevos Soles en fumar $84,6 \%$ (121alumnos) y más de $S / 5$ nuevos soles en porcentajes inferiores 15,4\% (22alumnos).

Factores personales: El consumo de droga es bajo $1,4 \%$ (2alumnos) en gran porcentaje no consumen droga 98,6\% (141alumnos). De la misma forma, el consumo de alcohol, se observan unas cifras un poco más elevadas que la droga $24,5 \%$ (35alumnos) y el factor que le induce a fumar, es la falta de práctica de deporte 44,1\% (63alumnos) (Tabla 1 ).
Tabla 1.Factores que influyen en el hábito de fumar.

\begin{tabular}{lcc}
\hline \multicolumn{1}{c}{ FACTORES } & $\mathbf{N}^{\circ}$ & $\mathbf{( \% )}$ \\
\hline A) FACTORES SOCIO & & \\
CULTURALES & & \\
Motivo & & \\
Curiosidad & 83 & 58,0 \\
Presión de grupo & 24 & 16,8 \\
Independencia & 10 & 7,0 \\
Rebeldía & 5 & 3,5 \\
Publicidad & 4 & 2,8 \\
Presencia de fumadores en la & 6 & 4,2 \\
familia & & \\
Estrés & 1 & 0,7 \\
Tensión por el estudio & 5 & 3,5 \\
Entorno familiar & 2 & 1,4 \\
Tristeza por estar lejos de la familia & 3 & 2,1 \\
& &
\end{tabular}

\section{B) FACTORES ECONÓMICOS}

\section{Gasto en fumar}

Menos de S/5.00 nuevos soles $\quad 121 \quad 84,6$

De 5 a 10 Nuevos Soles $\quad 15 \quad 10,5$

De 11 a 20 Nuevos Soles $\quad 3 \quad 2,1$

Más de 20 Nuevos Soles $\quad 4 \quad 2,8$

Monto asignado.

$\begin{array}{lcc}\text { Semanal } & 11 & 7,7 \\ \text { Mensual } & 21 & 14,7 \\ \text { Anual } & 111 & 77,6\end{array}$

$\begin{array}{lcc}\text { C) FACTORES PERSONALES } & & \\ \text { Consumo de droga } & & \\ \mathrm{Si} & 141 & 98,6 \\ \text { No } & & \\ \text { Factor que le induce a fumar } & 63 & 44,1 \\ \text { Falta de práctica de deporte } & 37 & 25,9 \\ \text { Fracaso y/o abandono familiar } & 43 & 30,1 \\ \text { Conflictos familiares } & & \end{array}$

El 26,93\% tiene el hábito de fumar, siendo el grupo etáreo predominante entre 16 a 20 años 62,2\% (89alumnos), el sexo predominante es el femenino $79,7 \%$ (114), estado civil soltero $97,4 \%$ (139), lugar de procedencia cercado de Ica $57,3 \%$ (82) siendo la edad que empezó a fumar 15 años a más $65 \%$ (93alumnos) con una frecuencia de fumar ocasionalmente $62,2 \%$ (89alumnos). (Tabla 2). 
Tabla 2. Características demográficas que influyen en el hábito de fumar.

\begin{tabular}{lcc}
\hline CARACTERISTICAS & $\mathrm{N}^{\circ}$ & $(\%)$ \\
\hline EDAD & & \\
16 a 20 & 89 & 62,2 \\
21 a 25 & 50 & 35,0 \\
26 a 30 & 1 & 0,7 \\
31 a 35 & 3 & 2,1 \\
SEXO & & \\
Masculino & 29 & 20,3 \\
Femenino & 114 & 79,7 \\
ESTADO CIVIL & & \\
Soltero & 139 & 2,8 \\
Casado & 4 & 97,2 \\
PROCEDENCIA & & \\
Cercado & 82 & 57,3 \\
Provincia & 40 & 28,0 \\
Otro departamento & 21 & 14,7 \\
EDAD QUE EMPEZÓ A FUMAR & & \\
10 años & 5 & 3,5 \\
11 años & 12 & 8,4 \\
12 años & 17 & 11,9 \\
13 años & 65 & 4,2 \\
14 años & 10 & 7,0 \\
15 años a más & 93 & 65,0 \\
FRECUENCIA DE FUMAR & & \\
Diario & 1 & 0,7 \\
Interdiario & 3 & 2,1 \\
A la semana & 7 & 62,2 \\
De vez en cuando & 43 & \\
En ocasiones & 89 & \\
\hline
\end{tabular}

\section{DISCUSIÓN}

En los últimos 20 años, el número de publicaciones e investigaciones sobre el comportamiento de fumar entre enfermeros, estudiantes y docentes de enfermería ha sido creciente fuera de América Latina. De la misma manera ocurre según revisión de la literatura en estudiantes universitarios de Lima, que un porcentaje considerable $(32,2 \%)$ fuman una vez a la semana(6).
En nuestro medio (Ica) el estudio señaló la ocurrencia mostrando el consumo de tabaco en cifras importantes un $26,93 \%$, teniendo que replantear medidas preventivas promocionales para establecer líneas de mejoramiento antitabaquismo tendientes a disminuir este porcentaje. Siendo influido por diversos Factores: Socioculturales, evidenciando el estudio que los estudiantes son motivados a fumar en su mayoría $58 \%$ (83 alumnos) por curiosidad, seguido por 
presión de grupo, por independencia y presencia de fumadores constituyó cifras $16,8 \%, \quad 7 \%$ y $4,2 \%$ respectivamente coincidente con Sánchez, quien señala que los jóvenes que fuman por primera vez lo hicieron por curiosidad en su mayoría, encontrándose en una mínima parte por la presión de amigos. La influencia de consumo de tabaco dentro de la familia, se encontró que fue por parte de los hermanos (10). Sin embargo, otro estudio en México mostró que el comportamiento de fumar fue influenciado por los medios de comunicación, principalmente la televisión en un 100\% (36 alumnos)(11), contrario a nuestro estudio ejecutado que la publicidad representó 2,8\% (4 alumnos), a nivel nacional (Perú), indicaron que el tener padres, hermanos o amigos que fuman, así como la opinión de que es definitivo o probablemente fácil dejar de fumar una vez que se ha empezado, constituyeron factores asociados al consumo corriente de tabaco en los estudiantes universitarios estudiados (7), coincidente este factor prevalente el consumo de cigarrillo y alcohol por familiares dentro del hogar, o sea influenciado por el entorno familiar señalaron el estudio efectuado por Castillo et al (8). En este contexto se señala que el consumo de tabaco sigue siendo un problema de salud pública, no solo por la magnitud de las consecuencias sanitarias, sino principalmente por la gran repercusión económica y social que genera.

Singularmente referente a los Factores económicos el monto utilizado para el consumo del tabaco ocasional es mínimo, no consistente al monto utilizado en Chile donde el $90 \%$ fuman entre 5 a 9 cigarrillos diario, con un costo económico mayor que los que fuman ocasionalmente (12). Aunque el estudio revela que un gran porcentaje fuman esporádicamente, sin embargo, el contacto con el tabaco y los efectos que causan en la salud, es un riesgo de salud pública que debe tomarse en cuenta para orientar a la población universitaria, evitando el consumo de cigarrillos que repercute en la economía familiar. En lo que se refiere a los Factores personales el estudio evidenció el consumo de drogas en cifras muy bajas 1,5\% (2alumnos) y a su vez estos fuman ocasionalmente pero la quinta parte de los estudiantes manifiestan beber licor $(24,5 \%)$ (35alumnos) y en su mayoría son los que fuman, casi la mitad de los fumadores beben alcohol y fuman. Observándose en otro estudio en Lima, Perú año 2002, porcentajes mayores del consumo de drogas $31,8 \%$. En relación al consumo del tabaco, asociado a otras drogas, el $42,8 \%$ de los fumadores afirmaron que fuman más cuando beben alcohol o usan drogas (7). Otro de los factores personales que influyó en nuestro estudio en el hábito de fumar fue en porcentajes significativos casi la mitad $(44,1 \%)$ (63alumnos) fue por falta de práctica de deporte, cerca del $30 \%$ fue por fracaso o abandono familiar y por conflictos personales. En este sentido se puede decir que la actividad deportiva es como un factor protector contra el hábito de fumar. 
Estudios como el de Holmen et al año 2002 en Noruega, establecen que la actividad deportiva se correlaciona de forma inversa con el hábito tabáquico de los adolescentes, encontrándose una correlación positiva entre el ejercicio y la función pulmonar en los que nunca habían sido fumadores (13). Se remarca que el consumo tanto del tabaco, alcohol y droga son perjudiciales para la salud por los efectos que ocasiona y las consecuencias desencadenantes posteriores a una posible dependencia, asociado a la concentración de nicotina en el fumador. Respecto a las características demográficas, el grupo etáreo predominante en el estudio efectuado fue 16 a 20 años $(62,2 \%)$, sexo femenino mayoritario, consistente con el hallazgo en Chile, que señalaron que la mayoría de los alumnos de la carrera de enfermería presentaron el hábito de fumar tienen entre 18 a 20 años(12). De la misma forma los resultados a nivel de Lima, Perú mostraron en su estudio que 6 de cada diez estudiantes tienen edades entre 16 y 22 años (2047 estudiantes participantes)(7).

En lo que concierne a la edad de inicio el estudio mostró que fue a los 15 años a más $(93 \%)$ y ocasionalmente, muy similar a estudios reportados en Colombia que señalan que alguna vez han fumado, lo hicieron por primera vez a los 15 años (14).

Asimismo se pudo revisar la literatura que en la mayoría de países así como en el nivel local (Ica), consistente con la edad de inicio que mostró nuestro estudio, pero en Lima, Perú se observó el inicio a muy temprana edad 12 años el 86,7\% (7). Siendo la edad uno de los factores más importantes que se debe tener en cuenta sobre todo considerando el inicio temprano en este hábito del tabaco, dirigiendo la atención supervisando en la educación primaria e inicio de la educación secundaria, enfatizando con medidas preventivas de las causas y efectos adversos para la salud.

En cuanto a la frecuencia de fumar, el estudio mostró que en ocasiones, mayoritariamente $62,2 \%$ (89 alumnos), el hábito diario es casi nulo $0,7 \%$ (1alumno) con una prevalencia de vida del tabaco de $26,93 \%$ consistente con el estudio en Sevilla que mostró el $26,1 \%$ año 2008 (15), porcentajes inferiores mostró en Asunción año 2007 señalando la prevalencia de tabaquismo de 16,8\% (población de estudiantes de enfermería y obstetricia)(16). Resultados superiores al estudio efectuado mostraron en Colombia el 39,5\%(14) cifras aún mayores en el mismo país Lima, Perú, donde la prevalencia de vida del tabaco fue $81,9 \%$ (7). Como se puede observar en nuestro estudio efectuado, el porcentaje de fumador diario es mínimo, pero ocasionalmente es una gran mayoría, no siendo de gran magnitud el consumo del hábito de tabaco, consuetudinario, pero es un problema que debe tomar en cuenta los organismos encargados de los programas de bienestar universitario.

\section{CONCLUSIONES}

Hay factores socioculturales, económicos y personales que influyen en el hábito de fumar en jóvenes universitarios, el estudio mostró 
un porcentaje significativo $26,93 \%$ superior a otro efectuado en la misma localidad (Ica) pero en alumnos de secundaria, cuya prevalencia del consumo fue $12,68 \%(8)$. Porcentajes aún mayores al nuestro $76,0 \%$ se revisó a nivel nacional (Lima, Perú)(7) así como en Colombia 39,5\%(14). Considerando que el consumo de Tabaco es un problema de salud pública que ocasiona graves riesgos para la salud, y que son los profesionales de la salud, los responsables de orientar a la población universitaria, estableciendo líneas de mejoramiento del cuidado a través de la implementación de estrategias preventivas antitabaquismo a nivel primario - secundario que es donde inicia la curiosidad por este hábito, contribuyendo a mejorar los estilos de vida saludable, libre de tabaco.

\section{Correspondencia:}

Dra. Isabel N. Urure Velazco

Correo electrónico: isabelnauv@yahoo.com

\section{REFERENCIAS BIBLIOGRAFICAS}

1. Guindon G, Boisclair D. Past, current and future trends in tabacco use [en línea] Washington: World Bank (Internet) 2003. (Citado 11 setiembre 2012) Disponible en:

http:/www.worldbank.org/tabacco/pdf/guin on-past $\% 2$ ocurrent-\%onhole.pdf

2. Hernández S, Guevara L, García M, Tascon J. Hábito de fumar en los estudiantes de Primeros Semestres de la Facultad de Salud: Características y percepciones. 2006; 37(1).

3. Organización Mundial de la Salud. Convenio Marco de la OMS para el control del tabaco. Lima: SINCO Editores; 2005. p.38.
4. Valdez R, Micher J.M, Hernández L, Hernández M. Tendencia del consumo de Tabaco entre alumnos de nuevo ingreso a la Universidad Nacional Autónoma de México. Salud Pública Mex. 2002; 44(S1):544 - 553.

5. Zavaleta A, Castro De La Matta R. Epidemiología de las drogas en la población urbana Peruana. 2003.Lima: Centro de información educación para la prevención contra el abuso de drogas; 2004. p. 96.

6. Comisión Nacional para el desarrollo y vida sin drogas. Mesa de trabajo de Universidades para la prevención de consumo de drogas. Encuesta sobre información, hábitos y actitudes hacia el consumo de sustancias psicoactivas en Universitarios de Lima Metropolitana. 2005. Lima: Comisión Nacional para el desarrollo y vida sin drogas (DEVIDA); 2005. p.37-41.

7. Zárate $M$, Zavaleta $A$, Danjoy $D$, et al. Prácticas de consumo del tabaco y otras drogas en estudiantes de Ciencias de la Salud de una Universidad Privada de Lima, Perú. Invest educ enferm. 2006;24(2):72-81.

8. Castillo R, Pastor N, Saravia N. Factores asociados al consumo de alcohol y Tabaco en adolescentes varones del centro educativo San Luis Gonzaga de Ica 2009-2010. Ica, Perú: Centro Educativo San Luis Gonzaga de Ica; 2010.

9. Peruga A. Tabaco: Lo que todos debemos saber. Washington D.C.: OPS/OMS 2002. 32: 3-32.

10. Díaz L, Sánchez E. Factores Psicosociales que presentan los jóvenes universitarios en el consumo del tabaco. Tesis. Veracruz, México. Facultad de Enfermería. Universidad Veracruzana; 2008.

11. Vasquez $F$, Pillon S, Cuamatzi $M$. Percepción de los estudiantes de Enfermería en cuanto al comportamiento 
de fumar en México. Texto \& Contexto enferm. 2004; 13(2): 203-208.

12. Robles L, Bobadilla A,Henriquez E. Factores que influyen en el hábito de fumar de los estudiantes de Enfermería universidad Santo Tomas sede Concepción Chile. Tesis. Facultad de Enfermería. Universidad Santo Tomás Concepción, Chile; 2008.

13. Holmen TL, Barret-Connor E, Clausen J, Holmen J,Bjermer L. Physical exercise, sports, and lung function in smoking versus non-smoking adolescents. Eur Respir Journal. 2002; 19:8-15.

14. Rodríguez M, Pineda $S$, Veles MF. Características del consumo de tabaco en estudiantes de enfermería de la Universidad de Antioquía. Tesis. Antioquía, Colombia. Facultad de Enfermería, Universidad de Antioquía, 2010.
15. García A, Nuñez S, Moreno A, et al. Evolución del Hábito Tabáquico en jóvenes universitarios en la primera década del siglo XXI. Tesis. Sevilla, España. Universidad de Sevilla, 2010.

16. Morel de FestnerJC. Conocimientos, actitudes y prácticas sobre el Tabaquismo en estudiantes de Enfermería y Obstetricia del Instituto $\mathrm{Dr}$ Andrés Barbero- Año 2007. Mem Inst Investig Cienc Salud. 2008;4(2):38-47.

Recibido: 02/11/13

Aprobado para Publicación: 30/11/13 